\title{
Formar Profesores de Educación Física para la Justicia Social: Efectos del Aprendizaje-Servicio en Estudiantes Chilenos y Españoles
}

\section{Training Physical Education Teachers for Social Justice: Effects of Service-Learning on Chilean and Spanish Students}

\author{
Luis García-Rico ${ }^{1 *}$ \\ Bastian Carter-Thuillier 2,3 \\ M. Luisa Santos-Pastor ${ }^{1}$ \\ L. Fernando Martínez-Muñoz ${ }^{1}$ \\ ${ }^{1}$ Universidad Autónoma de Madrid, España \\ ${ }^{2}$ Universidad de Los Lagos, Chile \\ ${ }^{3}$ Universidad Católica de Temuco, Chile
}

\begin{abstract}
Este artículo compara los efectos de dos modelos de Aprendizaje-Servicio en Educación Física para la formación de docentes desde una perspectiva de Justicia Social. Con un enfoque cuantitativo no experimental comparativo, la muestra se compone de 90 estudiantes universitarios de dos universidades: chilena y española que han participado en experiencias de Aprendizaje-Servicio en el ámbito de la Educación Física durante los cursos 2018/19 y 2019/20. El tratamiento y análisis estadístico se realizó mediante el programa SPSS v.25, estableciendo el nivel de significación para todos los análisis en $\mathrm{P}<0,05$. Los resultados del estudio muestran diferencias significativas sobre los efectos que produce cada modelo de AprendizajeServicio en la formación inicial desde una perspectiva de Justicia Social, utilizado en cada universidad. Asimismo, los resultados se encuentran en línea con otros estudios que muestran cómo la participación en experiencias de Aprendizaje-Servicio favorece el desarrollo de la reflexibidad crítica sobre la Justifica Social en los futuros docentes de Educación Física; además de otorgarles herramientas para la resolución de eventos problemáticos, a partir de situaciones comunitarias reales.
\end{abstract}

Descriptores: Educación física; Justicia social; Universidad; Chile; España.

This article compares the effects of two Service-Learning models in Physical Education for teacher training from a Social Justice perspective. With a quantitative approach, comparative non-experimental design, the sample is made up of 90 university students from two universities: Chilean and Spanish, who have participated in Service-Learning experiences in the field of Physical Education. Statistical analysis and processing was performed using the SPSS v.25 program, set the significance level for all analyzes at $\mathrm{P}<0.05$. The results of the study show significant differences on the effects produced by each Service-Learning model in initial training from a Social Justice perspective, used in each university. Equally, the results are in line with other studies that show how participation in Service-Learning experiences favors the development of critical reflexivity on Social Justification in future Physical Education teachers; in addition to granting them tools for the resolution of problematic events, based on real community situations.

Keywords: Physical education; Social justice; University; Chile; Spain.

*Contacto: luis.garciarico@gmail.com

ISSN: 2254-3139

www.rinace.net/riejs/

revistas.uam.es/riejs
Recibido: $\quad 20$ de mayo 2020

$1^{\text {a }}$ Evaluación: 13 de julio 2020

$2^{\text {a }}$ Evaluación: 19 de septiembre 2020

Aceptado: $\quad 3$ de octubre 2020 


\section{Revisión de la literatura}

Durante las últimas décadas, en diferentes regiones del planeta, la brecha de desigualdad social entre los sectores más desfavorecidos y aquellos que ostentan situaciones de privilegio se han acentuado de forma preocupante (Bayón y Saraví, 2019; Hurst, Fitz y Nurse, 2017; Nussbaum, 2012). Esta realidad ha demostrado incidir significativamente en el acceso al bienestar individual y social de un importante sector de la población (Mikucka, Sarracino y Dubrow, 2016). Así, resulta alarmante el hecho de que, en varios países, exista una creciente asimetría en la distribución de los ingresos (Palma, 2019), pero también en el reconocimiento y participación de las personas en la sociedad (Murillo y Hernández, 2011). En este sentido, Latinoamérica es precisamente uno de los contextos donde existen mayores índices de desigualdad, inequidad y segregación social (Lustig, 2020), situación que ha sido asimilada con normalidad durante años (Motta, Jelin y Costa, 2018), condicionando de forma importante el desarrollo y la convivencia en las fragmentadas sociedades latinoamericanas (Saraví, 2019). Este escenario ha puesto en tela de juicio a las propias democracias, sobre todo en lo que respecta a principios de justicia y equidad social (Bayón y Saraví, 2019; Sen, 2010).

Esta situación ha supuesto importantes consecuencias sobre los sistemas escolares y universitarios latinoamericanos (Fernández y Pérez, 2016; Gentili, 2015). De hecho, la realidad social está afectada por el propio sistema económico, político y cultural, y aunque sean campos interdependientes, se relega a un segundo plano la dimensión educativa (Carter-Thuillier y Moreno, 2017). Esto ha significado en múltiples ocasiones que los sistemas educativos acaben reflejando las situaciones de inequidad y marginación que existen con mayor notoriedad en las sociedades latinoamericanas (Tedesco, 2017; Tiramonti, 2016). Igualmente, implica que la escuela y las instituciones de Educación Superior acaben convirtiéndose en espacios con serias dificultades para subvertir situaciones de segregación y exclusión social, como las anteriormente mencionadas (Daude, 2012). Este escenario, además de ser preocupante, hace evidente también la necesidad de buscar urgentemente alternativas que permitan reconfigurar el papel del sistema educativo, con el objetivo de avanzar hacia realidades educativas centradas en la Justicia Social (Ramírez, 2016).

En el caso específico de Chile, se puede mencionar que diversos autores (Flores et al., 2019; García y Pérez, 2017; Nussbaum, 2012; Repetto, 2016; Solimano, 2017) se han referido a los altos índices de desigualdad que existen en el país, situación por la que la Organización para la Cooperación y el Desarrollo Económico (OCDE) lo ha considerado uno de los territorios más desiguales de Latinoamérica y del mundo (OCDE, 2016). Asimismo, diferentes estudios (Chavez y Flores, 2017; Duk y Murillo, 2019; Silva-Peña, 2020; Valenzuela, Bellei y De Los Ríos, 2014) han sido categóricos a la hora de indicar que el sistema educativo chileno tiene severos problemas de segregación (en términos socioeconómicos, culturales, étnicos, etc.). De hecho, además de ofrecer casi nulas posibilidades de movilidad social (Castillo, 2016), no otorga a los sectores con menores ingresos de la población la posibilidad de acceder a centros educativos con estándares elevados de calidad (Barahona, Veres y Barahona, 2018).

Esta problemática acaba configurando un sistema educativo que reproduce de forma permanente la estructura social existente (Sánchez, 2019) en una lógica de "status quo" (Ojeda et al., 2014). Es decir, la educación en el contexto chileno acaba siendo considerada como cualquier otro "bien de consumo" ordinario, al que se puede acceder en función de 
los méritos económicos que cada persona o grupo específico pueda poseer (Atria, 2012). Si a esto último se suman los reiterados cuestionamientos hacia el sistema educativo chileno por la baja pertinencia cultural de su currículum (Turra-Díaz, 2012), la histórica marginación de los saberes de los pueblos originarios en la escuela (Beltrán y Pérez, 2018) y el escaso reconocimiento a las necesidades de dichos colectivos y la población inmigrante (Jiménez et al., 2017), es evidente que estamos en presencia de un contexto social y educativo que posee importantes brechas en términos de Justicia Social.

Este contexto genera también, por consecuencia lógica, la necesidad de formar docentes comprometidos críticamente con la justicia y la transformación social (Cutforth, 2000; Domangue y Carson, 2008; Silva-Peña, 2020). Todo ello con el fin de contribuir a la superación de las situaciones de desigualdad (Peña-Sandoval y Montecinos, 2016), sobre todo aquellas que atentan contra la dignidad humana, para así avanzar hacia prácticas centradas en la redistribución, el reconocimiento y la representación de todos los colectivos e individuos (Murillo y Hernández, 2014). En este sentido, también resulta fundamental buscar alternativas metodológicas que permitan desarrollar en el futuro profesorado la capacidad de comprender las situaciones problemáticas de la población, así como las competencias necesarias para trabajar en la superación de las mismas (Aramburuzabala y García, 2012).

En este sentido, diferentes autores (Álvarez et al., 2017; Aramburuzabala y García, 2012; Chiva-Bartoll, Capella y Pallarès, 2018; García y Benítez, 2014; García y Sánchez, 2017; Mayor y Rodríguez, 2016, 2017; Miller y Nendel, 2011, Vázquez, Liesa y Lozano, 2017; Watson et al., 2002) describen la efectividad del Aprendizaje-Servicio (ApS) durante la formación inicial del profesorado, por lo que aporta para: (a) el desarrollo de un compromiso hacia prácticas de Justicia Social; (b) la sensibilización hacia colectivos marginados o invisibilizados; (c) la capacidad de trabajar bajo un enfoque de acción local y comunitaria; (d) la adquisición de competencias socioprofesionales, ciudadanas y relacionales; (e) el desarrollo de valores ciudadanos; (f) la capacidad de implementar prácticas pedagógicas para la innovación social; (g) desarrollo de competencias profesionales para disminuir la exclusión social y las dinámicas segregacionistas y marginadoras; y (h) aprender a trabajar participativamente con el resto de la comunidad educativa. El impacto del ApS en todas estas dimensiones puede tener relación con que dicha metodología permita la convergencia de cuatro elementos fundamentales en un sólo proyecto (Michigan State University, 2015): (a) competencias académicas; (b) aprendizaje de contenidos de las asignaturas; (c) desarrollo de valores y actitudes favorables hacia el trabajo comunitario; y (d) adquisición de habilidades crítico-reflexivas.

A pesar de que la práctica del ApS en Educación Superior es relativamente reciente, existen algunos reportes positivos respecto a su implementación en la formación del profesorado (Torres y Hasbún, 2019). Sin embargo, tal y como indican Iyer, Carrington, Mercer y Selva (2018), una de las ventajas de esta metodología es que ya ha sido probada de forma reiterada en el contexto internacional con resultados positivos. De hecho, en el espacio iberoamericano existen abundantes evidencias al respecto. Por ejemplo, en España los estudios de revisión (Redondo-Corcobado y Fuentes, 2019) muestran que en la última década existen numerosas experiencias de ApS en la formación inicial del profesorado (Álvarez, Sureda-Negre y Comas-Forgas, 2015; Álvarez et al., 2017; Gezuraga y Malik, 2015), en las que se describen las virtudes de esta metodología, sobre todo en lo que respecta al desarrollo de competencias ciudadanas y sociales para la inclusión, el trabajo 
comunitario y el desarrollo de habilidades críticas para comprender diferentes realidades (Iyer et al., 2018).

En el caso específico de la Educación Física (EF), el estudio de Chiva-Bartoll y sus colaboradores (2019) muestra que el ApS es un medio efectivo para la construcción de aprendizajes, así como para el desarrollo de competencias sociales y otras habilidades docentes de carácter específico (Capella-Peris, Gil-Gómez y Chiva-Bartoll, 2020). Además, ofrece al futuro profesorado de $\mathrm{EF}$ la oportunidad de vivir experiencias educativas inclusivas bajo un marco metodológico que permite vincular la teoría y la práctica de forma operativa (Chiva-Bartoll, Capella-Peris y Salvador-García, 2020). En este sentido, la naturaleza teórica-práctica-reflexiva del $\mathrm{ApS}$ ha demostrado impactar positivamente en la comprensión holística del proceso de aprendizaje, permitiendo así el desarrollo del sentido crítico (Cutforth, 2000; Herold y Waring, 2018), lo que acaba repercutiendo positivamente en el compromiso y responsabilidad social del futuro profesorado (Santos, Martínez-Muñoz y Cañadas, 2018). Esta sinergia positiva entre ApS y EF puede tener relación, entre otras cosas, con las propias características de esta disciplina, que puede utilizar como recursos pedagógicos varias herramientas con una elevada capacidad de socialización y alto interés comunitario (deportes, juegos, danzas, etc.), facilitando así su uso con fines de inclusión social (Lamoneda, Carter-Thuillier y López-Pastor, 2019).

En este marco, numerosos estudios avalan la influencia positiva del ApS sobre diferentes dimensiones relacionadas con el desarrollo académico, profesional, personal y social del estudiante universitario (Opazo, Aramburuzabala y Mcllrath, 2019; Rodríguez, 2014) y su incidencia en la formación para la Justicia Social. En concreto, algunos estudios valoran la utilización del ApS en la formación inicial de los estudiantes universitarios del ámbito de la EF, destacando sus efectos sobre:

- Desarrollo académico: Capella, Gil y Martí (2014) exponen la incidencia positiva del ApS en EF sobre el rendimiento académico y el desarrollo cognitivo del alumnado, fruto de la aplicación práctica de los conocimientos teóricos en situaciones reales y del uso del pensamiento crítico y reflexivo para abordar la problemática social. De esta forma, el ApS favorece la adquisición de aprendizajes contextualizados y significativos (Chiva-Bartoll et al., 2020).

- Desarrollo profesional: Los estudios dirigidos a valorar el impacto de las experiencias de ApS sobre la orientación vocacional y profesional del alumnado destacan las posibilidades que ofrece el ApS para la adquisición de las competencias generales y específicas que capacitan al alumnado para el desempeño de su futura labor profesional, tales como el trabajo cooperativo, la capacidad de planificar y desarrollar sesiones de ejercicio físico (Capella et al., 2020; Santos-Pastor, Martínez-Muñoz y Cañadas, 2018; Short et al., 2019).

- Desarrollo personal: El trabajo en pro del bien común, la implicación personal y la participación activa con los problemas sociales refuerzan la identidad ética y personal del estudiante (Ruiz-Montero, Chiva-Bartoll, Salvador-García y González-García, 2020), mostrando efectos positivos sobre su compromiso y responsabilidad cívica hacia la inclusión de los grupos sociales en desventaja (Corbatón et al., 2015; Meyers, Lemons y Hock, 2014).

- Desarrollo social: El ApS es considerado una poderosa estrategia pedagógica para la promoción de acciones prosociales y la adquisición de valores como la 
empatía, la solidaridad y el respeto (Chiva-Bartoll, Lidon y Salvador-García, 2020), que favorecen el empoderamiento social del alumnado para hacer frente a las injusticias sociales con la convicción de ser capaces de promover cambios sociales (Ruiz-Montero et al., 2020; Sanders, Galindo y DeTablan, 2019).

En función de los antecedentes expresados, parece especialmente relevante desarrollar estudios que permitan analizar el impacto del ApS sobre la formación de profesores de EF en el contexto latinoamericano y europeo. En este sentido, Chile es un lugar potencialmente interesante para ello, sobre todo si se tienen en cuenta las particularidades sociales y culturales de dicha nación. De este modo, podrían contrastarse los resultados del ApS en EF en el contexto chileno con los de otros países. En virtud de lo anterior, el objetivo del presente estudio es comparar los efectos sobre la formación inicial del profesorado de EF que tienen dos modelos de ApS desarrollados, ambos enfocados desde la Justicia Social: (1) el modelo de una universidad privada situada en la región de la Araucanía (Chile) y (2) el modelo de una universidad pública ubicada en España, concretamente en la Comunidad Autónoma de Madrid.

\section{Método}

\subsection{Contextualización y descripción de los modelos de ApS en EF}

\section{Modelo ApS en EF UCT (Región de la Araucanía, Chile)}

La Universidad Católica de Temuco (UCT), ubicada en la Región de la Araucanía, ha sido pionera a nivel nacional en la institucionalización del ApS. Ello significa que todas las carreras de dicha universidad deben incorporar el uso de esta metodología en los procesos formativos (UCT, 2016). En este sentido, las características del ApS se ajustan coherentemente a las necesidades de la Araucanía, entendiendo que se trata de la región con mayores índices de pobreza del país, además de tener alta presencia de la etnia Mapuche, un creciente porcentaje de inmigrantes y un importante número de organizaciones sociales (Ministerio de Desarrollo Social y Familia, 2018). Por ello, el modelo de ApS de la UCT considera también las particularidades culturales de la región, resignificando epistemológicamente lo que se entiende por "Aprendizaje" y "Servicio" a partir de las cosmovisiones culturales que coexisten en dicho territorio.

En el caso concreto de la carrera de Pedagogía en Educación Física, dicha unidad académica ha implementado progresivamente prácticas de EF vinculadas a ApS en diferentes asignaturas desde el año 2016. Para elegir las asignaturas que mejor se ajustan al uso de esta metodología y definir la forma óptima para implementarla en la carrera, la universidad ha empleado un modelo de cinco etapas: (a) definir comisión de trabajo; (b) análisis curricular; (c) experiencia piloto; (d) rediseño; y (e) consolidación. Ello ha derivado en la intervención en tres asignaturas los últimos tres años: (a) Educación Física Especial y Adaptada (tercer año): focalizada en el desarrollo de competencias docentes, para trabajar en contextos vinculados a discapacidad, y en la que los socios comunitarios son entidades que trabajan con población infanto-juvenil diagnosticada con capacidades diferentes; (b) Desarrollo y Evaluación Motriz Aplicada (segundo año): centrada en el desarrollo de competencias para la comprensión y diseño de situaciones motrices para el aprendizaje, y en la que los socios comunitarios son diferentes unidades públicas de la ciudad, vinculadas con el ámbito deportivo escolar; y (c) Teoría y Metodología de los Juegos (segundo año): enfocada en el desarrollo de competencias para el diseño de 
propuestas motrices con sentido lúdico, y en la que los socios comunitarios son los mismos que en la asignatura anterior.

En este sentido, la UCT ha optado por definir equipos de tres actores, para trabajar en el monitoreo y conversión de las asignaturas a formato de ApS: profesor encargado del curso, director de carrera y representante unidad institucional encargada de ApS. Asimismo, es importante mencionar que en todas las asignaturas el modelo utilizado tiene cuatro fases: diagnóstico, diseño, implementación y evaluación.

Modelo ApS en EF Universidad Autónoma de Madrid (Comunidad Autónoma de Madrid, España)

El Departamento de Educación Física, Deporte y Motricidad Humana de la Universidad Autónoma de Madrid (UAM) desarrolla desde hace unos años diferentes experiencias de ApS en el ámbito de la EF. El proyecto comenzó con una propuesta piloto en el curso 2016/17 en la asignatura Actividades Físico-Deportivas en el Medio Natural, del Grado en Ciencias de la Actividad Física y el Deporte (CCAFYD) y el alumnado con discapacidad intelectual que cursa el Programa Promentor (programa para la Inclusión Laboral de Personas con Capacidades Diferentes, organizado por la Cátedra UAM-Prodis ${ }^{1}$ ). Posteriormente, se ha ido expandiendo a diversas asignaturas del Grado en CCAFYD y del Grado en Magisterio en Educación Primaria (Mención en Educación Física), al mismo tiempo que se ampliaron, a través del convenio marco firmado con el Ayuntamiento de Madrid, los contextos de intervención y las redes de colaboración con diversos colectivos desfavorecidos, como son: mujeres sin hogar (Fundación FACIAM ${ }^{2}$ ), personas en situación de asilo o refugio (Fundación $\mathrm{CEAR}^{3}$ ) o con jóvenes en riesgo (Fundación Tomillo ${ }^{4}$. El propósito de estos proyectos, apoyándonos en Murillo y Hernández (2011), es el de fomentar la Justicia Social mediante la promoción de la Actividad Física y el Deporte, desde tres perspectivas: como distribución de recursos (culturales y de ocio) vinculados con la práctica de actividad física y deporte; como reconocimiento y respeto a los colectivos que presentan situaciones de desigualdad (acceso a la práctica de la actividad física y deporte); y como recurso para facilitar la participación de estos colectivos de manera activa y equitativa (elegir y compartir práctica de actividad física y deporte) para lograr su inclusión social.

Las experiencias de ApS en EF son elegidas de forma voluntaria por el alumnado como opción de trabajo en las asignaturas mencionadas. Éstas consisten en elaborar un diseño de intervención basado en una necesidad detectada y pactado previamente con el docente y los colectivos receptores, para ser desarrolladas en colaboración con los socios comunitarios y en relación con el programa de la asignatura. Al inicio del semestre académico se realiza una sesión conjunta (asamblea inicial) en la que participan el

\footnotetext{
${ }^{1}$ Programa de formación destinado a jóvenes con discapacidad intelectual cuyo objetivo se orienta a la inclusión laboral (http://www.sindromedownvidaadulta.org/no19-febrero-2015/articulosn19/programa-promentor-uam-prodis/)

${ }^{2}$ Federación de Asociaciones y Centros de Ayuda a Marginados (Ver https://faciam.org/que-esfaciam/)

${ }^{3}$ CEAR (Comisión Española de Ayuda al Refugiado)

${ }^{4}$ La Fundación Tomillo es una entidad privada, sin ánimo de lucro, no confesional e independiente que nace en 1984 con el propósito de contribuir a la mejora social y al desarrollo de la persona (Ver https://tomillo.org).
} 
alumnado universitario, los docentes responsables y los socios comunitarios, sirviendo como punto de partida para el conocimiento de los colectivos-receptores, el intercambio de ideas y la detección de necesidades que orienten la toma de decisiones. Las fases en las que se concreta el modelo de ApS en EF de la UAM son: observación inicial-diagnóstico, diseño de un plan, intervención, evaluación de la intervención y exposición de las experiencias.

\subsection{Diseño de la investigación}

La investigación se aborda desde un enfoque cuantitativo. Concretamente, se plantea un estudio no experimental comparativo, teniendo en cuenta las condiciones naturales en las que se realiza la investigación y la dificultad de controlar las variables del contexto.

Variables e hipótesis de la investigación

La variable independiente de la investigación es el impacto del ApS en EF como enfoque educativo para la Justifica Social en la formación académica, profesional, personal y social. La variable dependiente es el modelo de ApS en EF desde un enfoque de Justicia Social implantado por las universidades participantes.

Con el propósito de atender al objetivo principal de la investigación, se plantean las siguientes hipótesis: la aplicación de diferentes modelos de ApS en EF desde un enfoque de Justicia Social producirá diferencias estadísticamente significativas $(\mathrm{p}<0,05)$ en la valoración de los estudiantes de cada programa, respecto al impacto del ApS como enfoque de Justicia Social sobre su formación académica (H1), profesional (H2), personal (H3) y social $(\mathrm{H} 4)$.

\section{Población y muestra seleccionada}

La muestra del estudio la componen 90 estudiantes que habían participado en experiencias de ApS en EF durante su formación inicial en diferentes asignaturas de las titulaciones (Chile y Madrid): Carrera de Pedagogía en Educación Física (UCT) y Grado en Ciencias de la Actividad Física y el Deporte (UAM). Las características de la muestra participante se detallan en el siguiente cuadro:

Cuadro 1. Características de la muestra

\begin{tabular}{llrrc}
\hline & & UAM & UCT & TOTAL \\
\hline $\mathrm{N}$ & & 45 & 45 & 90 \\
\hline \multirow{4}{*}{ Edad } & $<20$ años & 18 & 5 & 23 \\
& $20-24$ & 24 & 33 & 57 \\
& $25-29$ & 2 & 7 & 9 \\
\multirow{2}{*}{ Sexo } & $>30$ & 1 & 0 & 1 \\
& Mujer & 21 & 20 & 41 \\
& Hombre & 24 & 25 & 49 \\
\multirow{3}{*}{ Curso } & $1^{\mathbf{0}}$ & 18 & - & 18 \\
& $2^{\text {o }}$ & 27 & 16 & 43 \\
& $3^{\text {o }}$ & - & - & 17 \\
& $4^{\circ}$ & - & 12 & - \\
\hline
\end{tabular}

Fuente: Elaboración propia.

\section{Técnicas de recogida y análisis de datos}

Terminadas las experiencias de ApS en EF desarrolladas en cada universidad, se procedió al envío del cuestionario "Escala de Aprendizaje-Servicio en Actividad Física (E_ASAF)" 
(Santos-Pastor et al., 2020), difundido entre el alumnado a través de correo electrónico. Dicho cuestionario contempla siete dimensiones (41 ítems y una pregunta abierta): (1) Identificación-Contexto; (2) Aprendizaje; (3) Valor pedagógico; (4) Impacto; (5) Desarrollo profesional; (6) Competencias profesionales; y (7) Opinión. A excepción de las cuestiones de la primera dimensión y la pregunta final de carácter abierto, el resto de los ítems se valoran a través de una escala Likert de 5 puntos, siendo 1- Totalmente en desacuerdo y 5- Totalmente de acuerdo.

Los datos fueron recogidos durante el curso académico 2018/19 y 2019/20. El tratamiento y análisis estadístico se realizó mediante el programa SPSS v.25, estableciendo el nivel de significación para todos los análisis en $\mathrm{P}<0.05$. Para valorar la percepción del alumnado universitario sobre el impacto que ha tenido en su formación inicial la participación en proyectos de ApS en EF desde una perspectiva de Justicia Social, se calcularon la media y la desviación típica a través de un análisis descriptivo de frecuencias. Asimismo, para conocer si existen diferencias significativas en las variables estudiadas en función del modelo de ApS en EF vivenciado, se realizó un análisis inferencial mediante la prueba $\mathrm{T}$ de Student.

\section{Resultados}

En primer lugar se comprobó la fiabilidad de la escala de medida utilizada (E_ASAF) a través del coeficiente Alfa de Cronbach $(\alpha=0,917)$, pudiendo valorarse como buena, al ser superior a 0,9. Seguidamente, se realizó el análisis descriptivo calculando las medias y desviaciones típicas de los ítems que conforman las diferentes dimensiones de la escala.

Los resultados obtenidos, teniendo en consideración el total de la muestra $(\mathrm{N}=90)$, revelan que más del $50 \%$ de los ítems son valorados de forma excelente $(\mathrm{M}>4)$. El alumnado de la UAM destaca como ítems mejores valorados los siguientes: (27) Recomendaría a mis compañeros hacer ApS ( $\mathrm{M}=4,76$; DT = 0,52); (16) Durante esta experiencia, fue enriquecedor trabajar con personas diferentes a mí $(\mathrm{M}=4,71$; DT $=0,54)$; y (35) Considero que es importante tener habilidades sociales y relacionales en los contextos de ApS $(\mathrm{M}=4,67$; DT = 0,52). Por su parte, el alumnado de la UCT valora superlativamente los ítems: (16) Durante esta experiencia, fue enriquecedor trabajar con personas diferentes a mí $(\mathrm{M}=4,51$; DT $=0,69)$; (27) Recomendaría a mis compañeros hacer ApS $(\mathrm{M}=4,49 ; \mathrm{DT}=0,66) ; \mathrm{y}(14)$ Destacaría el valor pedagógico de la experiencia de ApS frente al académico $(\mathrm{M}=4,40 ; \mathrm{DT}=0,75)$. Existe coincidencia en dos de los tres ítems seleccionados como mejores valorados por los estudiantes de ambas universidades. Únicamente dos ítems del total presentan una valoración significativamente menor que la del resto $(\mathrm{M}<3)$, siendo los menos valorados en ambos programas: (28) El ApS me ha ayudado a tener clara la elección de mi profesión; y (29) El realizar ApS me ha ayudado a enfocar mi futuro profesional respecto a la actividad laboral. Asimismo, los estudiantes del programa $\mathrm{ApS}$ en EF de la UAM añaden un tercer ítem a la lista de los menos valorados: (26) Sigo colaborando con el contexto donde hice el Aprendizaje-Servicio.

Finalmente, conociendo el promedio de cada uno de los ítems, se analizó si existen diferencias en las valoraciones de los estudiantes procedentes de cada universidad, en función del modelo de ApS en EF vivenciado. Para ello, se realizó la prueba T de Student para muestras independientes. El análisis comparativo muestra que 14 de los 31 ítems 
analizados presenta diferencias estadísticamente significativas. Dichas diferencias se abordan desde cada una de las dimensiones establecidas por el cuestionario (cuadro 2).

Cuadro 2. Comparación de resultados de los programas de APS-EF de UCT y UAM

\begin{tabular}{|c|c|c|c|c|}
\hline & & UAM & UCT & $\boldsymbol{P}$ \\
\hline \multirow{8}{*}{ 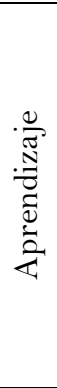 } & $\begin{array}{l}\text { 10. La participación en la experiencia de ApS me ha } \\
\text { ayudado a comprender los contenidos de la materia }\end{array}$ & $\begin{array}{c}3,98 \\
(0,78)\end{array}$ & $\begin{array}{c}3,13 \\
(0,69)\end{array}$ & $0,000^{*}$ \\
\hline & 11. La participación en la experiencia de ApS me ha & 4,38 & 4,00 & \multirow{2}{*}{$0,025^{*}$} \\
\hline & ayudado a experimentar los contenidos de la asignatura & $(0,74)$ & $(0,82)$ & \\
\hline & $\begin{array}{l}\text { 12. He aprendido más los contenidos haciendo ApS en la } \\
\text { comunidad que en las sesiones de aula }\end{array}$ & $\begin{array}{l}4,18 \\
(0,74)\end{array}$ & $\begin{array}{l}3,02 \\
(0,72)\end{array}$ & $0,000^{*}$ \\
\hline & 13. La participación en ApS me ha permitido ser más & 4,08 & 4,39 & \multirow{2}{*}{0,050} \\
\hline & responsable y protagonista de mi propio aprendizaje & $(0,79)$ & $(0,68)$ & \\
\hline & 14. Destacaría el valor pedagógico de la experiencia de & 4,51 & 4,40 & \multirow{2}{*}{0,478} \\
\hline & ApS frente al académico & $(0,72)$ & $(0,75)$ & \\
\hline \multirow{8}{*}{ 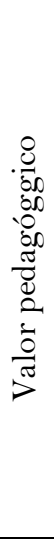 } & $\begin{array}{l}\text { 15. Desarrollé una buena relación con el profesorado que } \\
\text { me tuteló el ApS }\end{array}$ & $\begin{array}{c}4,36 \\
(0,74)\end{array}$ & $\begin{array}{c}3,96 \\
(0,82)\end{array}$ & $0,018^{*}$ \\
\hline & 16. Fue enriquecedor trabajar con personas diferentes a mí & $\begin{array}{c}4,71 \\
(0,54)\end{array}$ & $\begin{array}{c}4,51 \\
(0,69)\end{array}$ & 0,133 \\
\hline & $\begin{array}{l}\text { 17. El ApS me hizo consciente de algunos de mis propios } \\
\text { prejuicios }\end{array}$ & $\begin{array}{c}4,20 \\
(0,99)\end{array}$ & $\begin{array}{c}4,24 \\
(0,77)\end{array}$ & 0,813 \\
\hline & $\begin{array}{l}\text { 18. El aprendizaje-servicio me hizo superar ciertos } \\
\text { prejuicios }\end{array}$ & $\begin{array}{c}4,27 \\
(1,00)\end{array}$ & $\begin{array}{c}4,36 \\
(0,83)\end{array}$ & 0,649 \\
\hline & 19. El ApS me ayudó a reforzar mis habilidades de & 4,00 & 3,73 & \multirow{2}{*}{0,125} \\
\hline & liderazgo & $(0,97)$ & $(0,61)$ & \\
\hline & $\begin{array}{l}\text { 20. El ApS me ayudó a reforzar mis habilidades de trabajo } \\
\text { en equipo }\end{array}$ & $\begin{array}{l}4,47 \\
(0,75)\end{array}$ & $\begin{array}{l}4,07 \\
(0,65)\end{array}$ & $0,009^{*}$ \\
\hline & $\begin{array}{l}\text { 21. El ApS me ha permitido adaptar mis conocimientos al } \\
\text { contexto }\end{array}$ & $\begin{array}{c}4,47 \\
(0,62)\end{array}$ & $\begin{array}{c}4,31 \\
(0,66)\end{array}$ & 0,257 \\
\hline \multirow{6}{*}{ 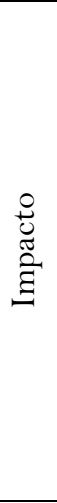 } & $\begin{array}{l}\text { 22. Siento que el trabajo desempeñado benefició a la } \\
\text { comunidad }\end{array}$ & $\begin{array}{c}4,44 \\
(0,62)\end{array}$ & $\begin{array}{c}4,31 \\
(0,63)\end{array}$ & 0,317 \\
\hline & $\begin{array}{l}\text { 23. El ApS me ha permitido conocer cómo involucrarme } \\
\text { profesionalmente en mi comunidad }\end{array}$ & $\begin{array}{l}4,44 \\
(0,58)\end{array}$ & $\begin{array}{c}3,84 \\
(0,67)\end{array}$ & $0,000^{*}$ \\
\hline & $\begin{array}{l}\text { 24. Siento que el trabajo desempeñado me hizo aprender } \\
\text { mi profesión }\end{array}$ & $\begin{array}{c}4,49 \\
(0,58)\end{array}$ & $\begin{array}{c}4,18 \\
(0,80)\end{array}$ & $0,040^{*}$ \\
\hline & $\begin{array}{l}\text { 25. Siento que el ApS me ha ayudado a valorar el interés de } \\
\text { mi servicio a la comunidad }\end{array}$ & $\begin{array}{c}4,33 \\
(0,64)\end{array}$ & $\begin{array}{c}3,96 \\
(0,63)\end{array}$ & $0,006^{*}$ \\
\hline & 26. Sigo colaborando con el contexto donde hice el ApS & $\begin{array}{c}2,82 \\
(1,23)\end{array}$ & $\begin{array}{c}4,04 \\
(0,79)\end{array}$ & $0,000^{*}$ \\
\hline & 27. Recomendaría a mis compañeros/as hacer ApS & $\begin{array}{c}4,76 \\
(0,52)\end{array}$ & $\begin{array}{c}4,49 \\
(0,66)\end{array}$ & $0,038^{*}$ \\
\hline \multirow{4}{*}{ 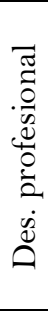 } & $\begin{array}{l}\text { 28. El ApS me ha ayudado a tener clara la elección de mi } \\
\text { profesión }\end{array}$ & $\begin{array}{c}2,65 \\
(0,83)\end{array}$ & $\begin{array}{c}2,99 \\
(0,97)\end{array}$ & 0,264 \\
\hline & $\begin{array}{l}\text { 29. El realizar ApS me ha ayudado a enfocar mi futuro } \\
\text { profesional respecto a la actividad laboral }\end{array}$ & $\begin{array}{c}2,74 \\
(1,02)\end{array}$ & $\begin{array}{c}2,97 \\
(1,15)\end{array}$ & 0,449 \\
\hline & $\begin{array}{l}\text { 30. El ApS debería ser una experiencia formativa } \\
\text { obligatoria en la carrera universitaria }\end{array}$ & $\begin{array}{c}4,00 \\
(1,26)\end{array}$ & $\begin{array}{c}4,22 \\
(0,76)\end{array}$ & 0,316 \\
\hline & 31. Integraré el ApS en mi profesión & $\begin{array}{c}3,73 \\
(1,09)\end{array}$ & $\begin{array}{c}3,80 \\
(0,99)\end{array}$ & 0,763 \\
\hline \multirow{4}{*}{$\begin{array}{l}+\stackrel{+}{0} \\
0 \\
0 \\
0 \\
0 \\
0 \\
0\end{array}$} & $\begin{array}{l}\text { 32. El ApS me ha hecho más consciente de las } \\
\text { competencias que se requieren para ser un buen } \\
\text { profesional }\end{array}$ & $\begin{array}{c}4,56 \\
(0,58)\end{array}$ & $\begin{array}{c}4,29 \\
(0,66)\end{array}$ & $0,046^{*}$ \\
\hline & $\begin{array}{l}\text { 33. Con el ApS he aprendido a resolver situaciones reales } \\
\text { similares a las que encontraré en mi profesión }\end{array}$ & $\begin{array}{c}4,36 \\
(0,77)\end{array}$ & $\begin{array}{c}4,18 \\
(0,57)\end{array}$ & 0,220 \\
\hline & $\begin{array}{l}\text { 34. El ApS me ha permitido saber planificar la } \\
\text { intervención y adecuarla a las necesidades del contexto }\end{array}$ & $\begin{array}{c}4,58 \\
(0,62)\end{array}$ & $\begin{array}{c}4,13 \\
(0,75)\end{array}$ & $0,003 *$ \\
\hline & $\begin{array}{l}\text { 35. Considero que es importante tener habilidades sociales } \\
\text { y relacionales en los contextos de ApS }\end{array}$ & $\begin{array}{l}4,67 \\
(0,52)\end{array}$ & $\begin{array}{l}4,33 \\
(0,69)\end{array}$ & 0,306 \\
\hline
\end{tabular}




\begin{tabular}{|c|c|c|c|c|}
\hline & $\begin{array}{l}\text { 36. Gestionar el grupo y contextualizar el aprendizaje es } \\
\text { fundamental en las experiencias de ApS }\end{array}$ & $\begin{array}{c}4,58 \\
(0,58)\end{array}$ & $\begin{array}{l}4,13 \\
(0,72)\end{array}$ & $0,002 *$ \\
\hline & $\begin{array}{l}\text { 37. Mi ApS estuvo relacionado con las competencias } \\
\text { profesionales }\end{array}$ & $\begin{array}{c}4,07 \\
(0,83)\end{array}$ & $\begin{array}{l}4,09 \\
(0,76)\end{array}$ & 0,896 \\
\hline & $\begin{array}{l}\text { 38. Tras la experiencia de ApS me siento competente para } \\
\text { generar cambios en la comunidad }\end{array}$ & $\begin{array}{l}4,09 \\
(0,70)\end{array}$ & $\begin{array}{c}3,82 \\
(0,65)\end{array}$ & 0,065 \\
\hline \multirow{3}{*}{ 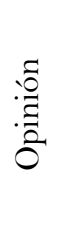 } & $\begin{array}{l}\text { 39. El ApS debería ser una actividad obligatoria en vez de } \\
\text { voluntaria }\end{array}$ & $\begin{array}{c}3,58 \\
(1,28)\end{array}$ & $\begin{array}{c}3,78 \\
(0,99)\end{array}$ & 0,413 \\
\hline & 40. El ApS debería de ser aplicado en otras asignaturas & $\begin{array}{c}4,49 \\
(0,72)\end{array}$ & $\begin{array}{c}4,11 \\
(0,77)\end{array}$ & $0,019^{*}$ \\
\hline & $\begin{array}{l}\text { 41. Me gustaría participar en otras experiencias de ApS en } \\
\text { la universidad }\end{array}$ & $\begin{array}{l}4,38 \\
(0,74)\end{array}$ & $\begin{array}{c}4,29 \\
(0,89)\end{array}$ & 0,610 \\
\hline
\end{tabular}

\section{Dimensión de aprendizaje}

Respecto a la dimensión de aprendizaje, existen diferencias significativas en la repercusión que tiene el ApS en EF sobre la comprensión de los contenidos teóricos y la posibilidad de aplicarlos en la práctica. Así, mientras que los estudiantes de la UAM manifiestan que la experiencia vivenciada les ha permitido aprender mejor los contenidos específicos de la materia que en las sesiones de aula, para el alumnado de la UCT no ha tenido el mismo efecto, pues se muestran imparciales ante dicha cuestión.

\section{Valor pedagógico}

En cuanto al valor pedagógico, se aprecian diferencias en el plano de las relaciones sociales que construyen los estudiantes de cada universidad con el docente que les tuteló durante el proyecto. Asimismo, se muestran diferencias sobre la contribución de las experiencias en el desarrollo de las habilidades que capacitan al alumnado a trabajar en equipo. Por el contrario, se aprecia una alta similitud en la valoración sobre el potencial del ApS en EF para concienciar y ayudar a superar ciertos prejuicios sociales. Esta valoración aporta claridad sobre el efecto del ApS en la consecución de objetivos de una formación inicial sensible a la Justicia Social.

\section{Impacto}

La dimensión sobre el impacto que producen las experiencias de ApS en EF en la comunidad es la que mayores diferencias presenta. Éstas versan sobre dos ámbitos: la vinculación profesional con el entorno y el compromiso de colaboración con los socios comunitarios. Respecto al primer ámbito, el alumnado de la UAM se destaca y distancia de los estudiantes de la UCT en su valoración sobre cómo la experiencia de ApS le permite involucrarse profesionalmente en la comunidad, reportándole aprendizajes sobre su profesión y concienciándole del interés del servicio prestado a los colectivos desfavorecidos. En el segundo ámbito se da la mayor de las diferencias que presenta el estudio. Gran parte de los estudiantes de la UCT siguen colaborando con el contexto donde realizaron las experiencias de ApS en EF, en contraposición a lo que sucede con los estudiantes de la UAM.

\section{Desarrollo profesional}

La siguiente dimensión no presenta diferencias estadísticamente significativas. Sin embargo, resulta interesante que los dos ítems menos valorados por el alumnado 
pertenecen a la dimensión de desarrollo profesional. Según los resultados obtenidos, los estudiantes de ambos programas coinciden en que las experiencias de ApS en EF que han llevado a cabo durante su formación inicial no les ha ayudado a reafirmar la elección de su profesión ni a enfocar su futuro profesional.

\section{Competencias profesionales}

Respecto a las competencias profesionales, se aprecian diferencias en la adquisición y desarrollo de habilidades para la planificación, gestión y adecuación de la propuesta de trabajo a las características del contexto y a las necesidades de los socios comunitarios. En este sentido, destaca la valoración que otorgan los estudiantes de la UAM en comparación con los de la UCT a la influencia del ApS en el desarrollo de las competencias profesionales en EF.

\section{Proyección del ApS}

Por último, existen diferencias estadísticamente significativas entre los estudiantes de las universidades participantes en el estudio en relación a su opinión sobre si el ApS debería utilizarse en más asignaturas de la titulación de EF. Aunque los dos grupos de estudiantes opinan que sí deberían ser aplicadas en otras asignaturas, el alumnado UAM lo afirma con mayor contundencia.

\section{Discusión y conclusiones}

$\mathrm{Al}$ amparo de los resultados presentados, se exponen los principales hallazgos del estudio. Los diferentes modelos de ApS en EF analizados parecen incidir positivamente en distintos ámbitos de la formación inicial del alumnado, destacando el potencial que ostenta este enfoque metodológico sobre la formación profesional (Santos-Pastor, MartínezMuñoz y Cañadas, 2018) y la construcción de la identidad social (Ruiz-Montero et al., 2020) desde una perspectiva de Justicia Social (Paz-Maldonado, 2019). Respecto a la formación profesional, este estudio pone en valor la importancia de desarrollar habilidades sociales y relacionales para el ejercicio de la labor profesional asumiendo los principios de la Justicia Social. En cuanto a la construcción de la identidad social, las experiencias de ApS generan una relación simbiótica que permite a los estudiantes formarse profesionalmente en un contexto real, percibiendo las situaciones de desventaja y generar beneficios a la comunidad (Tapia, 2008). De este modo, a través del acercamiento y actuación responsable en la comunidad por parte del estudiantado con el propósito de mejorarla, se genera un sentimiento de pertenencia a la comunidad alimentado por los beneficios que produjo su intervención.

Con la finalidad de atender al objetivo principal de la investigación, abordaremos las hipótesis planteadas inicialmente. En relación a la primera de ellas (H1), se confirman las notorias diferencias entre los estudiantes de cada universidad. Mientras que al alumnado de la UAM las experiencias de ApS en EF le ha permitido aprender, comprender y contextualizar los contenidos específicos de la asignatura, para los estudiantes de la UCT este enfoque metodológico no difiere sobre la adquisición de aprendizajes que logran conseguir en las tradicionales sesiones de aula. De manera que solamente los resultados obtenidos por el programa ApS en EF de la UAM se muestran en sintonía con el estudio de Miller y Nendel (2011) y de Chiva-Bartoll y otros (2020), respecto al impacto que tienen estas experiencias sobre la formación académica del alumnado, al propiciar la adquisición de aprendizajes significativos. 
Con respecto al impacto en la formación profesional de los estudiantes de $\mathrm{EF}$ (H2), existen diferencias significativas entre ambos modelos de ApS en EF en relación al conjunto de competencias que debe adquirir el alumnado durante su formación inicial. En las competencias transversales, las diferencias aparecen en la capacidad de trabajo en equipo, mientras que en las competencias específicas se reflejan en el desarrollo de habilidades para planificar y gestionar acorde a las necesidades del contexto. A pesar de que existen diferencias significativas, los estudiantes de ambas universidades valoran positivamente la influencia de las experiencias de ApS en EF en el desarrollo de las competencias profesionales, lo cual coincide con las investigaciones de Short et al. (2019) y Capella-Perís y otros (2020).

Respecto a la repercusión de las experiencias sobre la construcción de la identidad personal del alumnado (H3), este estudio no presenta diferencias sobre su implicación personal y compromiso ético con la comunidad. Los resultados obtenidos ya han sido descritos por numerosos autores, entre ellos Corbatón et al. (2015) y Ruiz-Montero et al. (2020), quienes han mostrado el beneficioso impacto de las experiencias de ApS en EF sobre la autonomía y responsabilidad del alumnado con su propio aprendizaje, así como para la eliminación de los prejuicios sociales.

En lo relativo a la última hipótesis del estudio (H4), los resultados confirman las marcadas diferencias sobre la dimensión social. Un elevado número de estudiantes de la UCT manifiesta que sigue colaborando con el contexto donde llevó a cabo la experiencia de ApS en EF, mostrando el poder de esta metodología para impulsar el desarrollo de acciones solidarias (Chiva-Bartoll et al., 2020). Esta situación es opuesta a la que se produce en la UAM, donde escasos estudiantes mantienen su vinculación con los socios comunitarios. Sin embargo, manifiestan que el ApS les ha permitido valorar la necesidad y el interés de este tipo de experiencias para la comunidad, que puede generar una actitud crítica hacia los problemas sociales y aumentar su compromiso con la comunidad y la lucha contra las injusticias sociales (Sanders et al., 2019). Este resultado evidencia el interés del ApS para contribuir a la formación inicial para la Justicia Social (Silva-Peña, 2020), sensibilizando a los futuros docentes respecto a la necesidad de transformar la educación y promover una sociedad más justa y equitativa (Paz-Maldonado, 2019).

En definitiva, tres de las cuatro hipótesis planteadas muestran diferencias estadísticamente significativas ( $\mathrm{H}_{1}, \mathrm{H} 2$ y H4), de modo que la valoración del alumnado sobre el modelo ApS en EF de la UAM le posiciona como una estrategia metodológica eficaz para el desarrollo de los aprendizajes académicos y profesionales propios de su titulación. El modelo de ApS en EF de la UCT destaca por su vinculación con el contexto y su contribución al desarrollo de la conciencia y responsabilidad social del alumnado, manteniendo y prolongando en el tiempo su colaboración con la comunidad.

Dados estos resultados, se hace evidente el potencial del ApS para generar prácticas pedagógicas centradas en la Justicia Social durante la formación inicial del profesorado especialista en EF (Miller y Nendel, 2011). Así, a partir de los resultados de este estudio y la evidencia previa existente, se puede señalar que la participación en actividades de ApS favorece en los futuros docentes de EF el desarrollo de la reflexividad crítica, además de ofrecer espacios para la resolución de situaciones comunitarias problemáticas y reales (Watson et al., 2002). Esto no sólo favorece el aprendizaje académico del profesorado en formación, sino también su capacidad para comprometerse en la transformación de situaciones de inequidad, exclusión, segregación o invisibilización que afrontan diferentes 
colectivos e individuos. En este sentido, teniendo en cuenta que los elementos antes mencionados son fundamentales para la formación de docentes desde un enfoque de Justicia Social, se puede decir que el ApS es una metodología válida y coherente para avanzar en esa dirección.

El presente estudio no solo permite conocer y comparar dos modelos de ApS en EF utilizados en distintos continentes, sino también conocer cómo impacta el enfoque metodologico del ApS en la formación integral de los estudiantes de EF, preparándoles para ejercer de forma responsable la profesión docente en la comunidad, con un marcado sentimiento de Justicia Social. Por tanto, los resultados de este estudio pueden ser de interés para profesores de $\mathrm{EF}$, equipos directivos de las universidades y representantes de la unidad institucional encargada del ApS. Aun así, el estudio presenta ciertas limitaciones con respecto al tamaño de la muestra y las universidades participantes. La aplicación del cuestionario E_ASAF a una muestra mayor y más diversa permitiría valorar con mayor exactitud el impacto que tiene en el alumnado la participación en experiencias de ApS en el ámbito de la formación inicial en EF. Además, un acercamiento cualitativo a la realidad aportaría una mayor comprensión de los efectos del ApS-EF en la formación docente desde una perspectiva de Justicia Social y desde la visión de los protagonistas implicados.

\section{Agradecimientos}

Este trabajo se ha realizado gracias a la Convocatoria del programa de Becas Iberoamérica Santander Universidades e Investigación (Curso académico 2019/20), que se enmarca dentro del convenio de colaboración entre la Universidad Autónoma de Madrid y el Banco Santander. Asimismo, este estudio se enmarca en la Red de Investigación en AprendizajeServicio en Actividad Física y Deporte para la Inclusión Social (RIADIS), financiada por el Consejo Superior de Deportes (2019) (www.riadis.es). Igualmente, este estudio se enmarca en el proyecto financiado por el Ministerio de Ciencia e Innovación, I+D+i PID2019-105916RB-Ioo/AEI / 10.13039/501100011033.

\section{Referencias}

Álvarez, J., Martínez, M., González, H. y Buenestado, M. (2017). El aprendizaje-servicio en la formación del profesorado de las universidades españolas. Revista Española de Pedagogía, 75(267), 199-2 17. https://doi.org/10.22550/REP75-2-2017-02

Álvarez, O., Sureda-Negre, J. y Comas-Forgas, R. (2015). Environmental education in pre-service teacher training: A literature review of existing evidence. Journal of Teacher Education for Sustainability, 17(1), 72-85. https://doi.org/10.1515/jtes-2015-0006

Aramburuzabala, P. y García, R. (2012). El aprendizaje-servicio en la formación de maestros. Revista del CIDUI, 1, 1-15.

Atria, F. (2012). La mala educación: Ideas que inspiran al movimiento estudiantil de Chile. CIPER.

Barahona, P., Veres, E. y Barahona, M. (2018). Factores asociados a la calidad de la educación en Chile. Revista Internacional de Investigación en Ciencias Sociales, 14(1), 17-30. https://doi.org/10.18004/riics.2018.julio.017-030

Bayón, M. y Saraví, G. (2019). Desigualdades: Subjetividad, otredad y convivencia social en Latinoamérica. Desacatos, 59, 8-15. https://doi.org/10.29340/59.2046 
Beltrán, J. y Pérez, S. (2018). Factores que dificultan la relación educativa entre la educación escolar y el saber y conocimiento mapuche. Diálogo Andino, 57, 9-20. https://doi.org/10.4067/So7 19-26812018000300009

Capella-Peris, C. Gil-Gómez, J. y Chiva-Bartoll, O. (2020). Innovative analysis of service-learning effects in physical education: A mixed-methods approach. Journal of Teaching in Physical Education, 39, 102-110. https://doi.org/10.1123/jtpe.2019-0030

Capella, C., Gil, J. y Martí, M. (2014). La metodología del aprendizaje-servicio en la educación física. Apunts Educación Física y Deportes, $116(2)$, 33-43. https://doi.org/10.5672/apunts.2014-0983.es.(2014/2).116.03

Carter-Thuillier, B. y Moreno, A. (2017). Globalización económica, postmodernidad y sistema educativo: Contradicciones y alternativas desde una educación física crítica. Estudios Pedagógicos, 43(3), 103-117. https://doi.org/10.4067/S0718-07052017000300006

Castillo, M. (2016). Fronteras simbólicas y clases medias. Movilidad social en Chile. Perfiles Latinoamericanos, 24(48), 213-241. https://doi.org/10.18504/pl2448-009-2016

Chavez, A. y Florez, M. (2017). Desigualdad educativa y el neoliberalismo en la educación chilena. Innova Research Journal, 2(3), 167-175. https://doi.org/10.33890/innova.v2.n3.2017.220

Chiva-Bartoll, O., Capella-Peris, C. y Salvador-García, C. (2020). Service-learning in physical education teacher education: Towards a critical and inclusive perspective. Journal of Education for Teaching, 46, 17-34. https://doi.org/10.1080/02607476.2020.1733400

Chiva-Bartoll, O., Capella, C. y Pallarès, M. (2018). Investigación-acción sobre un programa de aprendizaje-servicio en la didáctica de la educación física. Revista de Investigación Educativa, 36(1), 277-293. https://doi.org/10.6018/rie.36.1.270581

Chiva-Bartoll, O., Lidon, M. y Salvador-García, C. (2020). Can service-learning promote social well-being in primary education students? A mixed method approach. Children and Touth Services Review, 111, art 5. https://doi.org/10.1016/j.childyouth.2020.104841

Chiva-Bartoll, O., Ruiz-Montero, P., Martín Moya, R., Pérez López, I., Giles Girela, J., GarcíaSuárez, J. y Rivera-García, E. (2019). University service-learning in physical education and sport sciences: A systematic review. Revista Complutense de Educación, 30(4), 1147-1164. https://doi.org/10.5209/rced.60191

Corbatón, R., Moliner, L., Martí, M., Gil, J. y Chiva, O. (2015). Efectos académicos, culturales, participativos y de identidad del aprendizaje-servicio en futuros maestros a través de la educación física. Profesorado. Revista de Currículum y Formación del Profesorado, 19(1), 281297.

Cutforth, N. J. (2000). Connecting school physical education to the community through servicelearning. Journal of Physical Education, Recreation E Dance, 71(2), 39-45. https://doi.org/10.1080/07303084.2000.10606002

Daude, C. (2012). Educación, clases medias y movilidad social en América Latina. Pensamiento Iberoamericano, 10, 29-48.

Domangue, E. y Carson, R. (2008). Preparing culturally competent teachers: Service-learning and physical education teacher education. Journal of Teaching in Physical Education, 27(3), 347367. https://doi.org/10.1123/jtpe.27.3.347

Duk, C. y Murillo, F. J. (2019). Segregación escolar y meritocracia. Revista Latinoamericana de Educación Inclusiva, 13(1), 11-13. https://doi.org/10.4067/So718-73782019000100011 
Fernández, N. y Pérez, C. (2016). La educación superior latinoamericana en el inicio del nuevo siglo. Situación, principales problemas y perspectivas futuras. Revista Española de Educación Comparada, 27, 123-148. https://doi.org/10.5944/reec.27.2016.15044

Flores, I., Sanhueza, C., Atria, J. y Mayer, R. (2020). Top incomes in Chile: A historical perspective on income inequality, 1964-2017. The Review of Income and Wealth, 66(2), 1-25. https://doi.org/10.1111/roiw.12441

García, C. y Pérez, P. (2017). Desigualdad, inflación, ciclos y crisis en Chile. Estudios de Economía, 44(2), 185-221. https://doi.org/10.4067/S0718-52862017000200185

García, M. y Benítez, R. (2014). Comprometiéndonos con “nuestra escuela”: Un proyecto de aprendizaje-servicio para una formación del profesorado inclusiva. Revista de Educación Inclusiva, 7(2), 68-83.

García, M. y Sánchez, L. (2017). El aprendizaje-servicio y el desarrollo de las competencias emocionales en la formación inicial del profesorado. Contextos Educativos, 20, 127-145. https://doi.org/10.18172/con.2991

Gentili, P. (2015). América Latina, entre la desigualdad y la esperanza: Crónicas sobre educación, infancia $y$ discriminación. Siglo XXI.

Gezuraga, M. y Malik, B. (2015). Orientación y acción tutorial en la universidad: Aportes desde el aprendizaje-servicio. Revista Española de Orientación y Psicopedagogía, 26(2), 8-25. https://doi.org/10.5944/reop.vol.26.num.2.2015.15213

Herold, F. y Waring, M. (2018). An investigation of pre-service teachers' learning in physical education teacher education: Schools and university in partnership. Journal Sport, Education and Society, 23(1) 95-107. https://doi.org/10.1080/13573322.2015.1127802

Hurst, C., Fitz, H. y Nurse, A. (2017). Social inequality: Forms, causes, and consequences. Routledge. https://doi.org/10.4324/9781315536859

Iyer, R., Carrington, S., Mercer, L. y Selva, G. (2018). Critical service-learning: Promoting values orientation and enterprise skills in pre-service teacher programs. Journal Asia-Pacific Journal of Teacher Education, 46(2), 133-147.

https://doi.org/10.1080/1359866X.2016.1210083

Jiménez, F., Aguilera, M., Valdés, R. y Hernández, M. (2017). Migración y escuela: Análisis documental en torno a la incorporación de inmigrantes al sistema educativo chileno. Psicoperspectivas, 16(1), 105-116. https://doi.org/10.5027/psicoperspectivas-Vol16-Issue1fulltext-940

Lamoneda, J., Carter-Thuillier, B. y López-Pastor, V. (2019). Efectos de un programa de aprendizaje servicio para el desarrollo de prosocialidad y actitudes positivas hacia la inmigración en educación física. Publicaciones, 49(4), 127-144. https://doi.org/10.30827/publicaciones.v49i4.11732

Lustig, N. (2020). Desigualdad y descontento social en América Latina. Nueva Sociedad, 286, 5361.

Mayor, D. y Rodríguez, D. (2016). Aprendizaje-servicio y práctica docente: Una relación para el cambio educativo. Revista de Investigación Educativa, 34(2), 535-552. https://doi.org/10.6018/rie.34.2.231401

Mayor, D. y Rodríguez, D. (2017). Aprendizaje-servicio: Una práctica pedagógica que promueve la participación del estudiantado para la mejora escolar y social. Revista Complutense de Educación, 28(2), 555-571. https://doi.org/10.5209/rev_RCED.2017.v28.n2.49623 
Meyers, C., Lemons, L. y Hock, C. (2014). Implementing service-learning: Best practices from agricultural leadership education. Journal of Higher Education Outreach and Engagement, 18(3), 159-161.

Michigan State University. (2015). Service-learning toolkit. Center for Service-Learning and Civic Engagement.

Mikucka, M., Sarracino, F. y Dubrow, J. (2016). When does economic growth improve life satisfaction? Multilevel analysis of the roles of social trust and income inequality in 46 countries, 1981-2012. World Development, 93, 447-459. https://doi.org/10.1016/j.worlddev.2017.01.002

Ministerio de Desarrollo Social y Familia. (2018). Resultados encuesta CASEN 2017. Observatorio Social.

Motta, R., Jelin, E. y Costa, S. (2018). Introduction. En E. Jelin, R. Motta y S. Costa (Eds.), Global entangled inequalities: Conceptual debates and evidence from Latin America (pp. 5-17). Routledge. https://doi.org/10.4324/9781138019263-1

Murillo, F. J. y Hernández, R. (2011). Trabajar por la justicia social desde la educación. REICE. Revista Iberoamericana sobre Calidad, Eficacia y Cambio en Educación, 9(4), 3-6.

Murillo, F. y Hernández, R. (2014). Liderando escuelas justas para la justicia social. Revista Internacional de Educación para la Justicia Social, 3(2), 13-32.

Miller, M. P. y Nendel, J. D. (2011). Service-learning in physical education and related professions. A global perspective. Jones and Bartlett Publishers.

Nussbaum, M. (2012). Crear capacidades: Propuesta para el desarrollo humano. Paidós. https://doi.org/10.2307/j.ctvt7x63g

OCDE. (2016). OECD inequality update 2016. Income inequality remains high in the face of weak recovery. OCDE.

Ojeda, R., Carter-Thuillier, B., Cresp, M. y Rojas, J. (2014). La dimensión socioeconómica como condicionante en el sistema educativo chileno y la formación del profesorado: Una mirada crítica. Revista Dilemas Contemporáneos: Educación, Política y Valores, 2(1), 1-21.

Opazo, H., Aramburuzabala, P. y McIlrath, L. (2019). Aprendizaje-servicio en la educación superior: Once perspectivas de un movimiento global. Bordón. Revistas de Pedagogía, $71(3)$, art 1. https://doi.org/15-23. 10.13042/Bordon.2019.03.0001

Palma, J. (2019). Why is inequality so unequal across the world? Cambridge Working Papers in Economics, 50(5), 1-52.

Paz-Maldonado, E. (2019). Editorial. Repensar la formación docente desde una perspectiva de Justicia Social. Revista Electrónica de Investigación Educativa, 21(4), 1-4.

Peña-Sandoval, C. y Montecinos, C. (2016). Formación inicial de docentes desde una perspectiva de justicia social: Una aproximación teórica. Revista Internacional de Educación para la Justicia Social, 5(2), 71-86. https://doi.org/10.15366/riejs2016.5.2.004

Ramírez, A. (2016). Repensar la inclusión social desde la educación: Algunas experiencias en América Latina. Revista Internacional de Educación para la Justicia Social, 5(1), 177-194.

Redondo-Corcobado, P. y Fuentes, J. (2019). La investigación sobre el aprendizaje-servicio en la producción científica española: Una revisión sistemática. Revista Complutense de Educación, 31(1), 69-82. https://doi.org/10.5209/rced.61836

Repetto, A. (2016). Crecimiento, pobreza y desigualdad: La vía chilena. Economía y Política, 3(1), $71-101$. 
Rodríguez, M. R. (2014). El aprendizaje-servicio como estrategia metodológica en la universidad. Revista Complutense de Educación, 25, 95-113. https://doi.org/10.5209/rev_RCED.2014.v25.n1.41157

Ruiz-Montero, P. J., Chiva-Bartoll, O., Salvador-García, C. y González-García, C. (2020). Learning with older adults through intergenerational service-learning in physical education teacher education. Sustainability, 12(3), 11-27. https://doi.org/10.3390/su12031127

Sánchez, A. (2019). Factores asociados a la calidad de la educación en Chile. Mundo de la vida, habitus y reproducción en el análisis de la segmentación social de la educación chilena. Ensayos Pedagógicos, 14(2), 17-27. https://doi.org/10.15359/rep.14-2.1

Sanders, M., Galindo, C. y DeTablan, D. (2019). Leadership for collaboration: Exploring how community school coordinators advance the goals of full-service community schools. Children E' Schools, 41, 89-100. https://doi.org/10.1093/cs/cd zOO6

Santos-Pastor, M. L., Martínez-Muñoz, L. F. y Cañadas, L. (2018). Actividades físicas en el medio natural, aprendizaje-servicio y discapacidad intelectual. Espiral. Cuadernos del Profesorado, 11(22), 52-60. https://doi.org/10.25115/ecp.v11i22.1917

Santos-Pastor, M. L., Cañadas, L., Martínez-Muñoz, L.F. y García-Rico, L. (2020). Diseño y validación de una escala para evaluar el aprendizaje-servicio universitario en actividad física y deporte. Educación XX1, 23(2), 67-93. https://doi.org/10.5944/educXX1.25422

Saraví, G. (2019). La desigualdad social en América Latina: Explicaciones estructurales y experiencias cotidianas. Encartes, 2(4), 70-87. https://doi.org/10.29340/en.v2n4.113

Sen, A. (2010). La idea de justicia. Taurus.

Short, V., Covey, J. A., Webster, L. A., Wadman, R., Reilly, J. y Hay-Gibson, N. (2019). Considering the team in team formulation: A systematic review. Mental Health Review Journal, 24, 11-29. https://doi.org/10.1108/MHRJ-12-2017-0055

Silva-Peña, I. (2020). Formación docente para la justicia social en un Chile desigual. En I. SilvaPeña, J. Diniz-Pereira y K. Zeichner (Eds.), Justicia social: La dimensión olvidada de la formación docente (en prensa). Editorial Mutante.

Solimano, A. (2017). Estrategias de desarrollo económico en chile: Crecimiento, pobreza estructural y desigualdad de ingresos y riqueza. Centro Internacional de Globalización y Desarrollo.

Tapia, N. (2008). Calidad académica y responsabilidad social: El aprendizaje-servicio como puente entre dos culturas universitarias. En M. Martínez (Ed.), Aprendizaje-servicio y responsabilidad social de las universidades (pp. 57-89). ICE-Octaedro.

Tedesco, J. (2017). Educación y desigualdad en América Latina y el Caribe. Aportes para la agenda post 2015. Perfiles Educativos, 39(158), 206-224.

https://doi.org/10.22201/iisue.24486167e.2017.158.58790

Tiramonti, G. (2016). Notas sobre la configuración de la desigualdad educativa en América Latina. Revista Internacional de Educación para la Justicia Social, 5(1), 163-176.

Torres, V. y Hasbún, B. (2019). La historia del aprendizaje servicio en Chile. En V. Pizarro y B. Hasbún (Eds.), Aprendizaje servicio en la educación superior chilena. CEA-FEN.

Turra-Díaz, O. (2012). Currículo y construcción de identidad en contextos indígenas chilenos. Educación y Educadores, 15(1), 81-95. https://doi.org/10.5294/edu.2012.15.1.5

UCT. (2016). Competencias genéricas para la formación de profesionales integrales. Ediciones UCT. 
Valenzuela, J., Bellei, C. y De Los Ríos, D. (2014). Socioeconomic school segregation in a marketoriented educational system. The case of Chile. Journal of Education Policy, 29(2), 217-241. https://doi.org/10.1080/02680939.2013.806995

Vázquez, S., Liesa, M. y Lozano, R. (2017). Recreos cooperativos e inclusivos a través de la metodología de aprendizaje-servicio. Revista Electrónica Interuniversitaria de Formación del Profesorado, 20(1), 173-185. https://doi.org/10.6018/reifop/20.1.213181

Watson, D. L., Hueglin, S., Crandall, J. y Eisenman, P. (2002). Incorporating service-learning into physical education teacher education programs. Journal of Physical Education, Recreation $\mathcal{F}^{\circ}$ Dance, 73(5), 50-54. https://doi.org/10.1080/07303084.2002.10607810

\section{Breve CV de los/as autores/as}

\section{Luís García-Rico}

Estudiante de Doctorado en Ciencias de la Actividad Física y el Deporte en la Universidad Autónoma de Madrid (UAM). Miembro del Equipo Investigador RIADIS. Miembro de la Asociación de Aprendizaje-Servicio Universitario $\operatorname{ApS}(\mathrm{U})$. Autor de diversas comunicaciones en Congresos nacionales e internacionales relacionadas con el aprendizaje-servicio. Co-autor de la Escala Aprendizaje-Servicio en Actividad Física (EASAF). Ha participado y participa en diferentes proyectos educativos que tienen como eje principal el aprendizaje-servicio en el ámbito de la actividad física y el deporte, desarrollados en la Universidad Autónoma de Madrid (UAM) y la Universidad Católica de Temuco (chile). Ha colaborado en el rediseño de los proyectos de aprendizaje-servicio en la Universidad Católica de Temuco. ORCID ID: https://orcid.org/0000-0002-44561155. Email: luis.garciarico@gmail.com

\section{Bastian Carter-Thuillier}

Profesor de Educación Física. Licenciado en Educación. Máster en Ciencias Sociales para la Investigación en Educación y Doctor en Investigación Transdisciplinar en Educación. Actualmente es Académico del Departamento de Educación de la Universidad de Los Lagos (Chile). Posee experiencia como docente en programas de pregrado y postgrado de distintas universidades nacionales y extranjeras. Asimismo, ha realizado más de 40 publicaciones científicas especializadas y 30 conferencias en eventos nacionales e internacionales. Es miembro permanente del observatorio nacional del deporte y asesor de diferentes organizaciones públicas y privadas en materias vinculadas con la educación y el deporte. ORCID ID: https://orcid.org/0000-0001-7342-4179. Email: bastiancarter@gmail.com

\section{Luisa Santos-Pastor}

Licenciada y doctora en Educación Física. Graduada en Educación Social. Profesora titular en el Departamento de Educación Física, Deporte y Motricidad Humana en la Universidad Autónoma de Madrid. Coordinadora del área de Innovación de la Cátedra Unesco de Educación para la Justicia Social de la UAM. Coordinadora del Grupo de Investigación UAM Enseñanza y Evaluación de la Actividad Física y el Deporte. Coordinadora de la Red de Investigación en Aprendizaje-Servicio en Actividad Física y el Deporte para la Inclusión Social. IP. Proyecto I+D " Aprendizaje-Servicio Universitario en Actividad Física y el deporte. Oportunidad para la inclusión social. (Ref. PID2019- 
105916RB-100). Líneas de investigación principales: Aprendizaje-Servicio en Actividad Física y Deporte; Actividades Física en el Medio Natural; Evaluación Formativa y compartida en Educación Superior; Metodologías Activas en Educación Física. ORCID ID: https://orcid.org/0000-0002-4985-0810. Email: marisa.santos@uam.es

\section{Fernando Martínez-Muñoz}

Doctor en Educación Física con especial interés en el campo educativo, (currículo, metodologías activas y evaluación formativa), así como en el desarrollo de las AFDMN en diferentes contextos (educación, ocio, salud). Desde el año 1998, ha desarrollado su labor docente en la Universidad de Almería (1998-2010) y Autónoma de Madrid (actualidad) como Profesor Contratado Doctor. Pertenece a la Red de Evaluación Formativa (2005), EF en la Naturaleza (2016) y ApS en AFD (2019). Es autor de diferentes publicaciones de impacto y ha participado en proyectos $\mathrm{I}+\mathrm{D}+\mathrm{i}(2001$, 2008, 2013 y 2020) sobre turismo activo, evaluación formativa y ApS-AFD. ORCID ID: https://orcid.org/0000-0001-5209-7527. Email: f.martinez@uam.es 